$\mathrm{AR}_{\text {EVIEW }}$

AUTHOR FOR CORRESPONDENCE :

CHANDRA SHEKHAR

Department of Veterinary Public

Health and Epidemiology, College

of Veterinary Science and Animal

Husbandry, N.D. University of

Agriculture and Technology,

FAIZABAD (U.P.) INDIA

Email: cshekharvph@gmail.com

\section{Ebola virus disease with special reference to epidemiology and management}

\author{
CHANDRA SHEKHAR
}

\begin{abstract}
Ebola virus disease (EVD) is a highly fatal viral zoonotic disease. The disease was first reported in Africa in 1976. Since then the disease outbreaks have occurred several times in different years. The disease is mainly found in African countries. The causative agent is Ebola virus which belongs to the family Filoviridae. The case fatality rate is around 50 per cent but in different outbreaks, it showed variations from 25 per cent to 90 per cent. Monkeys and chimpanzees are important reservoirs of Ebola virus. The virus is transmitted to people from wild animals. Ebola is introduced into the human population through close contact with the blood, secretions, organs or other bodily fluids of infected wild animals such as chimpanzees, gorillas, fruit bats, monkeys etc. Ebola then spreads through human-to-human transmission via direct contact with the blood, secretions, organs or other bodily fluids of infected people, and with surfaces and materials contaminated with these fluids. The disease is characterized by fever, headache and myalgia, followed by diarrhoea, vomiting, abdominal symptoms and dehydration. Collapse, neurological manifestations and bleeding particularly in gastrointestinal tract may occur in the last phase of the disease. Community awareness is important in controlling the outbreaks of disease. The disease management involves case management, surveillance and contact tracing, a good laboratory service, safe burials and social mobilization. Early supportive care with rehydration, symptomatic treatment improves survival of affected person. No licensed vaccines are available yet, but 2 potential vaccines are undergoing human safety testing. cAd3-ZEBOV is a chimpanzee derived adenovirus vector with an Ebola virus gene inserted. rVSV-ZEBOV is an attenuated vesicular stomatitis virus with one of its genes replaced by an Ebola virus gene. ZMapp is the best known emerging treatment so far. It is a combination of three humanised monoclonal antibodies targeted at three Ebola virus glycoprotein epitopes. Before the current 2014 outbreak, ZMapp had proved protective when given to non-human primates 24-48 hours after infection. TKM-Ebola is another drug consists of a combination of small interfering RNAs that target Ebola virus RNA polymerase L, formulated with lipid nanoparticle technology. It has been shown to be protective in non-human primates.
\end{abstract}

Key words : Ebola virus, Epidemiology, Current outbreaks, Transmission, Management

How to cite this paper : Shekhar, Chandra (2015). Ebola virus disease with special reference to epidemiology and management. Vet. Sci. Res. J., 6(2) : 103-112.

Paper History : Received : 01.05.2015; Accepted : 28.09.2015 